\title{
Directed Energy Interstellar Propulsion of WaferSats
}

\author{
Travis Brashears $^{(1)}$, Philip Lubin ${ }^{(1))}$, Gary B. Hughes ${ }^{(2)}$, Kyle McDonough ${ }^{(1)}$, Sebastian Arias ${ }^{(1)}$, \\ Alex Lang(1), Caio Motta ${ }^{(1)}$, Peter Meinhold ${ }^{(1)}$, Payton Batliner ${ }^{(1)}$, Janelle Griswold ${ }^{(1)}$, Qicheng \\ Zhang $^{(1)}$, Yusuf Alnawakhtha ${ }^{(1)}$, Kenyon Prater ${ }^{(1)}$, Jonathan Madajian ${ }^{(1)}$, Olivia Sturman ${ }^{(1)}$, \\ Jana Gergieva ${ }^{(1)}$, Aidan Gilkes ${ }^{(1)}$, Bret Silverstein ${ }^{(1)}$ \\ (1)Physics Department, University of Santa Barbara, CA 93106, trbrashears@ gmail.com, +1-805- \\ 893-8432 \\ ${ }^{(2)}$ Statistics Department, California Polytechnic State University, San Luis Obispo, CA 93407, \\ gbhughes@calpoly.edu, +1-805-756-5648
}

Keywords: Interstellar Travel, Interstellar Exploration, Directed Energy, Laser Sail, WaferSats

\begin{abstract}
In the nearly 60 years of spaceflight we have accomplished wonderful feats of exploration and shown the incredible spirit of the human drive to explore and understand our universe. Yet in those 60 years we have barely left our solar system with the Voyager 1 spacecraft launched in 1977 finally leaving the solar system after 37 years of flight at a speed of $17 \mathrm{~km} / \mathrm{s}$ or less than $0.006 \%$ the speed of light. As remarkable as this is, we will never reach even the nearest stars with our current propulsion technology in even 10 millennium. We have to radically rethink our strategy or give up our dreams of reaching the stars, or wait for technology that does not exist. While we all dream of human spaceflight to the stars in a way romanticized in books and movies, it is not within our power to do so, nor it is clear that this is the path we should choose. We posit a technological path forward, that while not simple; it is within our technological reach. We propose a roadmap to a program that will lead to sending relativistic probes to the nearest stars and will open up a vast array of possibilities of flight both within our solar system and far beyond. Spacecraft from gram level complete spacecraft on a wafer ("wafer sats") that reach more than $1 / 4 \mathrm{c}$ and reach the nearest star in 15 years to spacecraft with masses more than $10^{5} \mathrm{~kg}$ (100 tons) that can reach speeds of near $1000 \mathrm{~km} / \mathrm{s}$ such systems can be propelled to speeds currently unimaginable with our existing propulsion technologies. To do so requires a fundamental change in our thinking of both propulsion and in many cases what a spacecraft is. In addition to larger spacecraft, some capable of transporting humans, we consider functional spacecraft on a wafer, including integrated optical communications, optical systems and sensors combined with directed energy propulsion. Since "at home" the costs can be amortized over a very large number of missions. The human factor of exploring the nearest stars and exo-planets would be a profound voyage for humanity, one whose non-scientific implications would be enormous. It is time to begin this inevitable journey beyond our home.
\end{abstract}

\section{Introduction}

We propose a system that will allow us to take the step to interstellar exploration using directed energy propulsion combined with miniature probes including some where we would put an entire spacecraft on a wafer to achieve relativistic flight and allow us to reach nearby stars in a human lifetime.

With recent work on wafer scale photonics and directed energy, we can now envision combining these technologies to allow for a realistic approach of sending probes far outside our solar system and to nearby stars. By leaving the main propulsion system back in Earth orbit (or nearby) and propelling wafer scale highly integrated spacecraft that include cameras, bi-directional optical communications, power and other sensors we can achieve gram scale systems coupled with small laser driven sails to 
achieve relativistic speeds and traverse the distance to the nearest exoplanets in a human lifetime. While this is not the same as sending humans it is a step towards this goal and more importantly allows us to develop the relevant technological base and the ability to build a single "photon driver" to send out literally millions of low mass probes in a human lifetime as well as a mixture of very low mass and very high mass system as we will discuss. This technology is critical to radical reformulation of spaceflight for extremely high speed mission ranging from relativistic interstellar gram to kilogram probes to interplanetary travel allowing missions to Mars (for example) in days rather than years for human crewed craft. While we will focus most of the discussion in this document to relativistic ultra-low mass probes the ability for the same technology to be used in our solar system and beyond for very large spacecraft should be understood. The key to the system lays in the ability to build both the photon driver and the ultra-low mass probe. Recent developments now make this possible. We have outlined our directed energy propulsion system in [1] [2] [3] [4] [5] [6] [7]

\section{Scaling}

Since the system we propose is not single use but rather scalable to any size it is critical to understand

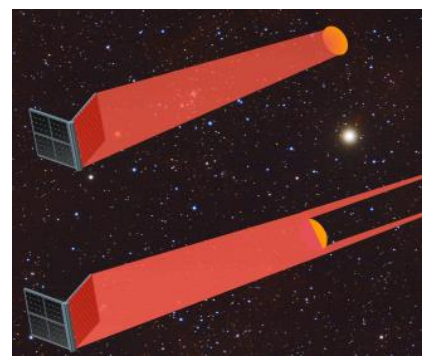

Figure 1. Conceptual drawing showing beam filling sail and then as distance increases eventually overflowing. With Continued illumination speed increases by $2^{1 / 2}$. the scaling relations in the section above. In general we use the optimized case of payload mass $=$ sail mass and assume a nearly ideal sail tuned to the laser wavelength so $\epsilon_{\mathrm{r}}=1$. We assume a slightly futuristic sail with thickness of $1 \mu \mathrm{m}$ for many cases and $10 \mu \mathrm{m}$ (thick even for todays sails). Future advancements in sails thickness down to $0.1 \mu \mathrm{m}$ and below can be envisioned but are NOT assumed. They will only make the conclusions even more optimistic. The density of all sails we consider is about the same, namely $\rho \sim 1,400 \mathrm{~kg} / \mathrm{m}^{3}$. Note the scaling of speed [2].

The scaling of speed is a mild function of payload mass $\sim \mathrm{m}_{0}{ }^{-1 / 4}$. This is due to the fact that as the payload mass grows so does the sail. As the sail grows the acceleration distance increases as the laser spot can become larger: $v_{\max -\infty}=\left(\frac{2 P_{0} d}{c \lambda}\right)^{1 / 2}\left(h \rho m_{0}\right)^{-1 / 4}$ which scales as $\mathrm{P}_{0}^{1 / 2}, \mathrm{~d}^{1 / 2}, \mathrm{~h}^{-1 / 4}, \rho^{-1 / 4}, \mathrm{~m}_{0}^{-1 / 4}$.

\section{Wafer Scale Spacecraft}

Recent work at UCSB on Si photonics now allows us to design and build a "spacecraft on a wafer". [8] The recent (UCSB) work in phased array lasers on a wafer for ground-based optical communications combined with the ability to combine optical arrays (CMOS imagers for example) and MEMS accelerometers and gyros as well as many other sensors and computational abilities allows for extremely complex and novel systems. [9] Traditional spacecraft are still largely built so that the mass is dominated by the packaging and interconnects rather than the fundamental limits on sensors. Our approach is similar to comparing a laptop of today to a super computer with similar power of 20 years ago and even a laptop is dominated by the human interface (screen and keyboard) rather than the processor and memory. Combining nano photonics, MEMS and electronics with recent UCSB work on Si nano wire thermal converters allows us to design a wafer that also has an embedded RTG or beta converter power source (recent LMCO work on thin film beta converters as an example) that can power the system over the many decades required in space. Combined with small photon thrusters (embedded LEDs/lasers for $\mathrm{nN}$ thrust steering on the wafer gives a functional spacecraft. While not 
suitable for every spacecraft design by any means this approach opens up radically new possibilities. In addition the power from the laser itself can add significant power to the spacecraft even at large distances. We have run link margin calculations including Zodi, CIB, galaxy and optical emission for a wafer scale laser communications system run in a hibernate/ burst mode using the DE-STAR array as both the transmitter of power to propel and communicate with the spacecraft as well as to receive the very weak signal from the spacecraft and conclude it is feasible to receive data (albeit at low rate) at light year distances. For pointing we use the wafer camera to star track and/or lock to DE-STAR laser as a beacon. [7]

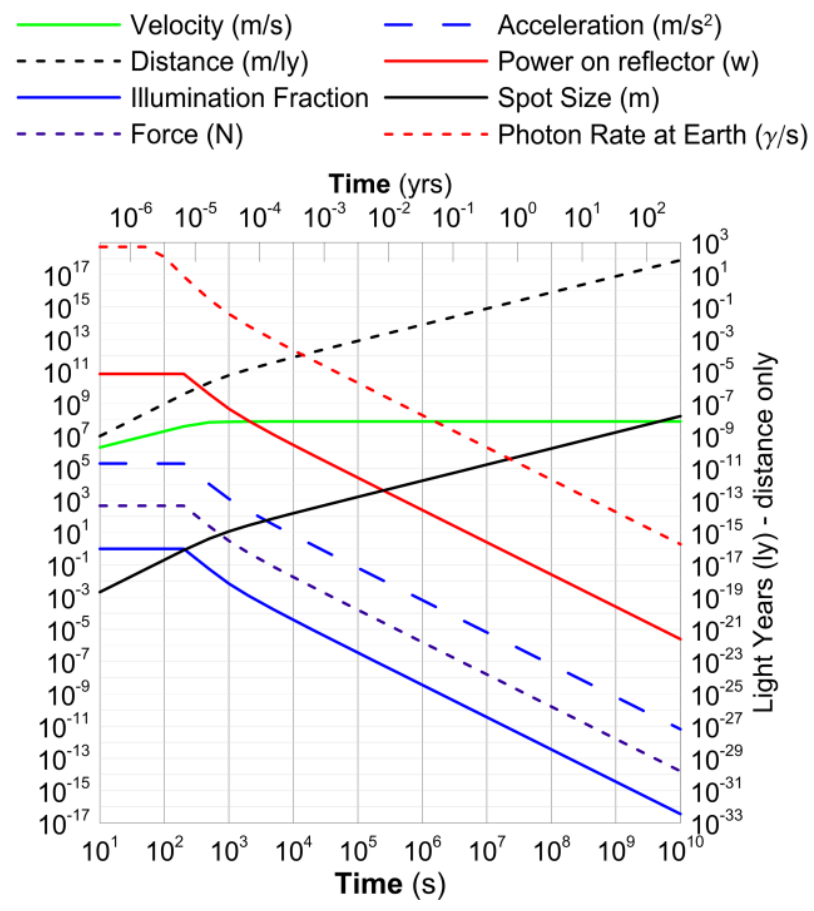

Figure 2. Parameters for full class 4 (70GW) system with wafer SC and $1 \mathrm{~m}$ sail. Craft achieves $26 \% \mathrm{c}$ in about $10 \mathrm{~min}$ and takes about 15 years to get to Alpha Centauri. Communications rate assumes class 4 drive array is also used for reception with a 1 watt short burst from a $100 \mathrm{~mm}$ wafer SC. Here the only optical system on the spacecraft is assumed to be the $100 \mathrm{~mm}$ wafer. No external optics is assumed. The data rate at Alpha Centauri with the wafer alone is about $0.35 \mathrm{kbs}$ during the 1 watt burst. Using the $1 \mathrm{~m}$ drive reflector as part of the optical comm system increases the data rate shown by a factor of 100 to about $35 \mathrm{kbs}$ during the 1 watt burst. Nominal optical comm duty cycle is about $0.5 \%$ (comm laser on) assuming a $5 \mathrm{mw}$ electrical RTG with current conversion efficiency $(6.5 \%)$ using a $\mathrm{Pu}$ source.

\section{Mission Design for reaching Alpha Centauri}

Our mission plan is designed to take full advantage of the scalability of our wafer scale craft. The wafer scale crafts, although incredibly fast, have limited payload mass and therefore limited utility as well. By sending many crafts of different sizes to Alpha Centauri we obtain the best of both worlds.

One of the key points of our mission design is the "shotgun" approach. Sending out many wafer scale craft at a range of sizes and instrumentation. The wafer sized crafts are fast but delicate. Contact with interstellar particulate could be catastrophic while moving at such high speeds. The best approach to deploying the wafer crafts is to launch many of them with varied instrumentation, as well as duplicates per each type of instrumentation. This approach insures that some spacecraft of each type make it to Alpha Centauri. The trade-off between speed and payload mass is at the core of our mission design. Since our ship design is scalable, we must consider all the ships that meet the requirements of this project. We will consider ships of the following masses in our mission plan: $1 \mathrm{~g}, 10 \mathrm{~g}, 100 \mathrm{~g}$, and $1 \mathrm{~kg}$. These ships can all make it to Alpha Centauri within 100 years. We also consider much larger spacecraft that are designed for very long-term interstellar travel. The "shotgun" approach will be applied not only for the wafer sized but for the rest as well. The electronics onboard each spacecraft 
are small enough to be assembled on earth and transported to space. The reflectors may have to be assembled in space, as the reflector for the $1 \mathrm{~kg}$ class ship is 27 meters in diameter.

Our spacecraft design features on-board power. This allows us to not only take data at Alpha Centauri, but along the way as well. Our Science Objectives and Instrumentation section touches on some of the possible measurements that could be done by our ships en-route to the Alpha Centauri star system. Once the ships breach the AC star system the true purpose of the mission begins. The wafer sized ships will be the first to reach the AC star system as they are by far the fastest ships. The wafers ships priorities will be to send back images of the Alpha Centauri binary star system, as well as search for exoplanets, measure the radiation fields, the magnetic field environment, and stellar winds around Alpha Centauri. Obviously the types of measurements taken at Alpha Centauri will vary with the instrumentation onboard each ship, however images of the star and detection of planets in the star system are a high priority (confirmation or denial of the existence of Alpha Centauri Bb- possible earth sized exoplanet).

The amount of time each ship spends passing through the Alpha Centauri star system varies depending on the speed of the ship. We will use $100 \mathrm{AU}$ (very roughly twice the distance from our sun to Pluto) as a "data taking zone", just to shed light upon relative time frames each class of probes has to make measurements, and to use as a feasible number for preliminary calculations. The wafer class ships have only about 55.2 hours in a "data taking zone" of this size, amounting to 993 bursts of data. The ten gram ship would spend around 98.5 hours, the hundred gram ship 197.1 hours, and the $1 \mathrm{~kg}$ ship 345 hours. Bound orbits around Alpha Centauri are not feasible at speeds this high, so all the ships will eventually pass through the star system and fan out into the great beyond. After leaving Alpha Centauri, all ships will continue to map the interstellar medium sending back noteworthy data. We do consider the case of using a MagSail in order to slow down the spacecraft to enter an orbit, but we do not know at this time if this is feasible.

\section{Laser Sail}

The laser sail is both similar to and fundamentally different than a solar sail. For small sails, even with low powers the flux can easily exceed $100 \mathrm{MW} / \mathrm{m}^{2}$ or $10^{5} \mathrm{Suns}$. This requires a very different approach to the sail. For the small reflectors we propose using a pure dielectric reflection coating on ultra-thin glass or other material. Spherical (bubbles) sails are an option for testing. The loss in fiber optic quality glasses allows loss in the $\operatorname{ppt}\left(10^{-12}\right) / \mu \mathrm{m}$ (of thickness) which is even better than we need. This is an area we need to explore much more. The flux at the tip of high power single mode fiber optic exceeds $10 \mathrm{TW} / \mathrm{m}^{2}$, higher than we need. Rather than the typical $1 / 4$ reduced wavelength anti reflective (AR) dielectric coating, we will need to design a 1/2 wave reflection coating for the sail. The scaling of flux on the reflector is:

Flux $=P_{0} / D^{2}$ Assume optimized case where sail mass = payload mass $m_{0}=m_{\text {sail }}=D^{2} h \rho$ $D=\left(m_{0} / h \rho\right)^{1 / 2}$ Flux $=P_{0} / D^{2}=P_{0} h \rho / m_{0}$

Note the flux is proportional to the thickness and density (smaller sail) and inversely proportional to the mass (larger sail). This means lower mass payloads have high flux requirements on the sail.

\section{Multi layer dielectric on metalized plastic film}


Metalized thin film plastic films with multi layer dielectric coatings can achieve very high reflectivity.

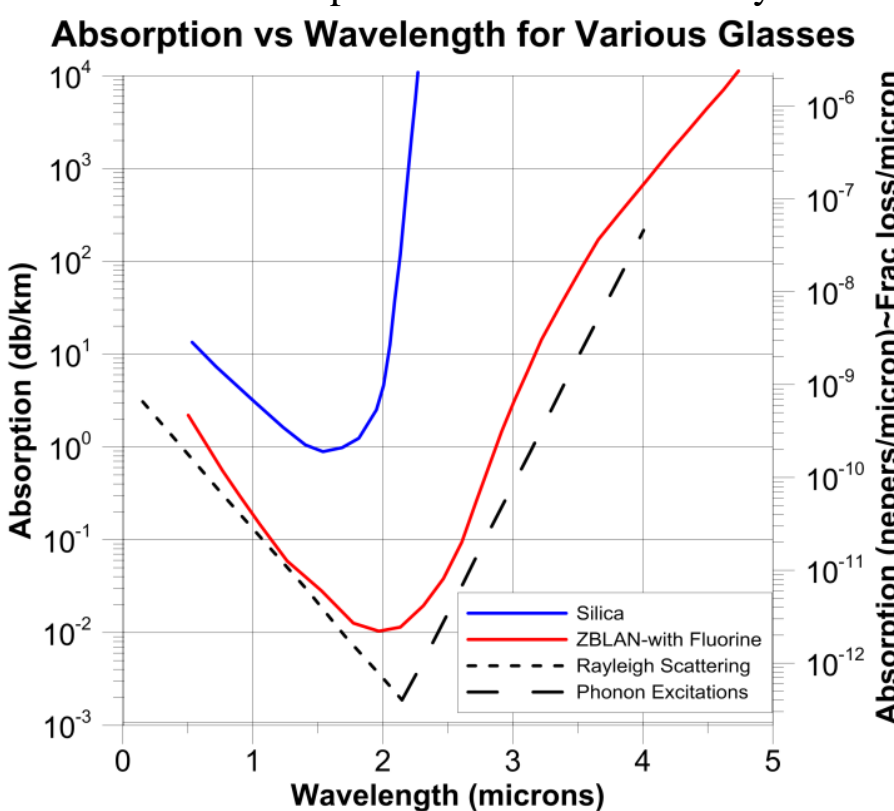

In collaboration with industry we have designed a $99.995 \%$ reflective system suitable for large scale "roll to roll" production. Note the reflectivity is tuned to the narrow laser line and that these reflectors are NOT suitable for solar sails which use the broad spectrum of the sunlight to propel them. We illustrate this below with a putative design for our $\mathrm{Yb}$ baseline $1064 \mathrm{~nm}$ laser case. For large sails (>10 $\mathrm{m}$ diameter) this is a suitable choice. For example a 30 meter square sail on $10 \mu \mathrm{m}$ thick plastic film will have a mass about $13 \mathrm{~kg}$ while a more advanced thin film of $1 \mu \mathrm{m}$ thickness would have a mass of about $1.3 \mathrm{~kg}$.

Figure 3 - Multilayer dielectric deposited on metalized plastic film. The reflectivity is tuned to be maximum at the laser wavelength. Left - Reflectivity vs wavelength for several models of the reflector with varying dielectric layers and compositions.

\section{Cloaking}

One worry that arises from our use of a dielectric film for our reflector is a direct cause of its low absorption. A large reason why our reflector would be able to achieve such low absorption is because not all light that is not reflected is absorbed. Some of the light that is not reflected is allowed to pass right through the reflector. This is a great benefit to us in regards to the reflectors absorptivity, but we run into a concern. Our wafer is designed to lie behind our reflector, hence in the direct path of the light that is allowed to go through our reflector. This light might have a negative impact on our wafer, and the instrumentation located on our wafer. A short and quick answer to the solution arises: fiber optics. As a solution to this concern, we propose using fiber optic cables to guide the light that traverse the reflector away from our wafer, therefore avoiding any damage that could possibly be done by the excess light that is not reflected or absorbed. This is the equivalent of a cloacking device as the laser does not "see" the wafer.

\section{Optical phased array for communication}

For the spacecraft's onboard communications system, we propose a small photonic phased array laser with a silicon-on-insulator (SOI) platform. Optical phased array technology is progressing rapidly. Members of the Electrical Engineering department at UCSB have demonstrated in a study that using an integrated approach to manufacturing an optical phased array (using a single photonic integrated circuit, rather than many individual optical components) can greatly reduce size, weight, and cost of the device by removing packaging for the individual components [9]. Benefits of the optical phased array include:

Free space beam steering. This allows the array to angle the outgoing beam within a range of angles in two directions from the normal. This is highly useful for tracking the receiving end of communications while the ship moves through space [9]. 
No moving parts. The communication device is much less susceptible to mechanical wear than its counterparts that also allow for free space beam steering.

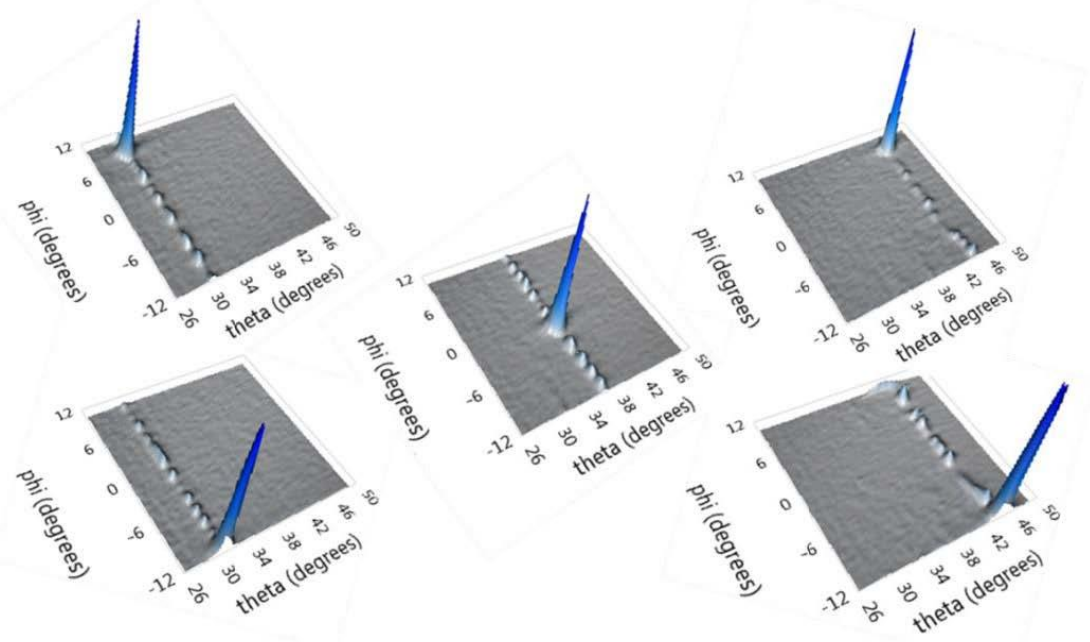

The hybrid silicon platform designed at UCSB allows for on-chip laser design. This means that the laser that feeds the phased array of waveguides is attached to the Silicon chip itself [9], which complements our ship-on-achip design. We will working with our ECE photonics groups at UCSB that are already designing and building laser comm phased arrays on a chip.

Figure 4. UCSB Phased array for chip level laser communication with no external optics showing electronic beam steering. Hulme et al., 2014.

\section{Spacecraft to DE-STAR ( $1 \mathrm{~m}$ reflector $\sim 2$ grams) data rate}

Using a DE-STAR 4 [18] (10 km array) as our satellite receiver on the Earth side, the divergence halfangle for a circular aperture of diameter $10 \mathrm{~cm}$ (nanophotonic phased array covering one side of the wafer), and optical communication wavelength of $1.06 \mu \mathrm{m}$, [10] we calculate

$\theta_{\text {transmission }}=1.22\left(\frac{\lambda}{\mathrm{D}}\right)=1.29 \times 10^{-5}$ radians $\quad \tan \left(\theta_{\text {transmission }}\right)=\left(\frac{\mathrm{R}_{\text {spot }}}{\mathrm{L}}\right)$

Where $\mathrm{R}_{\text {spot }}$ is the radius of the beam spot at the receiving end, and $\mathrm{L}$ is the distance from Earth to Alpha Centauri, 4.3 light years.

$\mathrm{R}_{\text {spot }}=\mathrm{L} \cdot \tan \left(\theta_{\text {transmission }}\right)=5.25 \times 10^{11} \mathrm{~m} \quad\left(\frac{\text { Area }_{\text {spot }}}{\text { Area }_{\text {receiver }}}\right)=\left(\frac{\text { Photons }_{\text {transmitted }}}{\text { Photons }_{\text {received }}}\right)=1.10 \times 10^{16}$

Single-photon-detecting devices exist and are expected to improve in the near future. The National Institute of Standards and Technology and NASA's Jet Propulsion Laboratory have developed a superconducting nanowire single-photon-detecting array (2 nanowires $\mathrm{x} 2$ nanowires) that obtains more than one bit per photon. Therefore our communication system must be capable of receiving at least one photon on the Earth side.

Current state-of-the-art laser technology converts electricity into laser power with $76 \%$ efficiency, and $80 \%$ is the stated goal of the DARPA Super-Efficient Diode Sources (SHEDS) program.

$$
\mathrm{E}_{\text {Photon }}=\left(\frac{\mathrm{hc}}{\lambda}\right)=1.88 \times 10^{-19} \mathrm{~J} \quad\left(1.88 \times 10^{-19} \mathrm{~J} / \text { photon }\right) \cdot\left(1.10 \times 10^{16} \text { photons }_{\text {transmitted }}\right)=2.07 \times 10^{-3} \mathrm{~J}
$$

Assuming 76\% efficiency, the single-photon-communication power requirement becomes: 
$\left(\frac{2.07 \times 10^{3} \mathrm{~J}}{0.76}\right)=2.72 \times 10^{3} \mathrm{~J}$.

Because $1 \mathrm{~W}=1 \mathrm{~J} / \mathrm{s}$, we see that communication at a rate of one bit (per photon) per second requires less than $3 \mathrm{~mW}$.

$$
\left(\frac{1}{2.72 \times 10^{-3} \mathrm{~W} / \text { photon }}\right) \cdot\left(\frac{1 \text { bit }}{\text { photon }}\right)=368 \text { bits } / \mathrm{W}
$$

We receive nearly 370 bits/s within a 1-Watt burst. We therefore submit that 1 -second 1-Watt bursts of power are sufficient in order to send data back from Alpha Centauri. $5 \mathrm{~mW}$ of onboard power generation will allow us 18 such bursts per hour or one about every 3 minutes. The reason to use a burst mode is purely to enhance to signal recovery. It is important to understand the average data rate is not the same as the burst data rate. The average data rate is given by $370 \mathrm{bit} / \mathrm{W} * 0.005 \mathrm{~W} \sim 2 \mathrm{bps}$. Generally spacecraft communicate via burst mode even in LEO or for interplanetary missions so this is not unusual. Using the reflector for laser communications would also help greatly. We have NOT assumed this in the link margin calculations above but is one of the options listed later on.

\section{Option of using drive reflector in optical data link}

By repositioning the WafeSat we could conceivably use the drive reflector as a mirror to greatly enhance the data rate sent back. Using the drive reflector as the transmit reflector increases our data rate at Earth by approximately 100x and thus get us to about $37 \mathrm{kbs}$ during the 1 watt burst mode at the distance of Alpha Centauri. The average data rate is about $200 \mathrm{bps}$. This is fast enough to allow compressed images of the star and any planets or extra solar small bodies (asteroid etc that might exist in the Alpha Centauir system) to be sent back as well as other sensor readings such as magnetic field strength, radiation environment etc. In addition we can use the same reflector as part of our imaging system. Note that at 1 ly ( 1/4 the way to Alpha Centauri) we get approximately $500 \mathrm{kbs}$ during data bursts. Thus we could get "live streaming" (modulo TOF) much of the way, The challenges of maintaining the shape of the reflector into a diffraction limited optic are formidable at the ultra-low masses we are trying to achieve for our fastest WaferSats. This is less challenging for our more massive missions architectures. There are many challenges here that will require considerable effort but the rewards will be used in not only interstellar probes but also planetary, space and terrestrial remote sensing, medical, security, etc. The list of possible uses for self-contained autonomous wafer scale systems is endless.

\section{DE-STAR to Spacecraft (1m reflector $\sim 2$ grams) data rate}

While receiving a signal does not actually require any local power, a signal amplifier (which does require local power) is typically used unless the incoming signal is very strong.

The power received at the spacecraft is calculated the same way as the power received at the satellite, using a $100 \mathrm{GW}$ optical transmission and a $10 \mathrm{~km}$ aperture:

$\theta_{\text {transmission }}=1.29 \times 10^{-10} \quad \mathrm{R}_{\text {spot }}=5.25 \times 10^{6}$ 


$$
\begin{aligned}
& \left(\frac{\text { Area }_{\text {receiver }}}{\text { Area }_{\text {spot }}}\right)=\left(\frac{\text { Power }_{\text {receiver }}}{\text { Power }_{\text {transmitted }}}\right)=1.15 \times 10^{-16} \mathrm{~W}_{\text {received }} / \mathrm{W}_{\text {transmitted }} \\
& 100 \mathrm{GW} \cdot 1.15 \times 10^{-16} \mathrm{~W}_{\text {received }} / \mathrm{W}_{\text {transmitted }}=1.125 \times 10^{-5} \mathrm{~W}=1.15 \times 10^{-5} \mathrm{~J} / \mathrm{s} \\
& \left(\frac{1.15 \times 10^{-5} \mathrm{~J} / \mathrm{s}}{1.88 \times 10^{-19} \mathrm{~J} / \text { photon }}\right)=6.12 \times 10^{13} \text { photons } / \mathrm{s}
\end{aligned}
$$

Assuming the same data rate of 1 photon/bit, this means we obtain over 60 Tbits/second. In general we do not have a need for such a rapid "uplink mode" except perhaps to reprogram the system. In all of this the time of flight must be factored in as it take more than 4 years for the signal to travel from Alpha Centauri to Earth.

\section{Instrument Requirements}

Obviously, power will be consumed by more than just our communications array. Instrumentation also requires power. One of the most important instruments for our probe is a spectrometer. Commercially available microspectrometers exist today that are $2.5 \mathrm{~cm}^{3}$, 'weigh' 5 grams, and consume $30 \mathrm{~mW}$ of power. Even better, a paper published in IEEE in 2002 described a CMOS optical microspectrometer that covered the visible range, had an area of less than $16.4 \mathrm{~mm}^{2}$, consumed only $1.25 \mathrm{~mW}$ of power, and could be used in concert as an array to increase the spectral range. Mass was not reported, but is assumed to be sub-gram. This existed 13 years ago. One of our groups at UCSB is a world leader in chip level photonics. We will be working with them to design a custom photonics and electronics

The second most likely instrument for our probe to carry is a magnetometer. In 2010, a research team at the University of Cincinnati produced a paper detailing the production of a micromagnetometer, which consumed only $14 \mathrm{~mW}$ and had a sensor area of $0.3 \mathrm{~mm}^{2}$. Mass was not reported, but is assumed to be sub-gram. Depending on the sensitivity level we desire we do not see an issue with a fully wafer scale instrument suite. Additionally, Geometrics has a roadmap for wafer-scale production of their (total field) Micro-Fabricated Atomic Magnetometers, and claims that a $1 \mathrm{~cm}^{3}$ version which consumes on the order of $200 \mathrm{~mW}$ will be available in 18 months. This is an extremely sensitive magnetometer and would be suitable to our larger payload systems.

The third instrument that we would like to carry on our probe is a camera. Digital Optics Corporation has developed MEMS camera technology that requires only $1 \mathrm{~mW}$ of power. Due to the popularity of smartphones, MEMS camera technology is an area in which we are likely to see significant and continuous improvement for the foreseeable future. See System Architecture write-up below on the Advancement of MEMS technology. Since we only need to take a picture infrequently the average power consumption for the imaging section of our system will be extremely small. As an example if we take 1 photo per hour (1440 per day) with a 60 second exposure (integration) time we will only have an average draw of 60/3600*1 mw $\sim 17 \mu \mathrm{W}$. We could easily reduce this by taking fewer images.

\section{Microcontroller}


We will implement an ultra-low power micro-controller to run the electronics on our probe. Currently existing (commercially available) micro-controller technology achieves $160 \mu \mathrm{A} / \mathrm{MHz}$ consumption in active mode and $10 \mathrm{nA}$ consumption in sleep mode with full memory retention. As our applications will also be relatively low-voltage, which will amount to a very small power draw and fits nicely within design constraints.

\section{Redundancy and Fault Tolerance}

A key element of our design will be redundant critical system elements and fault tolerance as well as watchdog elements. Due to the very long cruise phase and possibility for dust hits it will be critical to have multiple independent processors and sensors. The wafer is large enough (it has the capacity for more than $10^{12}$ devices with $14 \mathrm{~nm}$ processing) to allow at least 4 way redundancy on many elements. We may be able to have dual independent RTG power sources but this needs a full wafer design layout to validate. A first layout looks feasible to do this. For the larger systems (not single wafer) this is much easier.

\section{Redundancy through large scale launches}

Since our system allows for an extremely large number of spacecraft to be sent, (recall we can send a wafer scale system every 10-15 minutes) we also have massive redundancy from the sheer number of redundant spacecraft. For wafer scale systems once the NRE is done for a single wafer we can mass produce other wafers (spacecraft) thus radically reducing the costs per mission. This is a radical departure from traditional spacecraft designs which are "one off" with the NRE dominating the cost. For the smaller system we advocate mass production of spacecraft while the largest systems (1-100 tons) are much more like traditional spacecraft designs.

\section{Energy Storage}

In addition to the RTG power source we need to have a small energy storage system to handle the high power short duty cycle portions of the spacecraft. These include the laser communications (comm) system and some higher power sensors. The two current possibilities are capacitors (especially so called "super capacitors") and electrochemical cells. The primary issues are mass and lifetime. This is a very rapidly evolving area that is dramatically improving. The numbers we will quote are for the current state of the art (SOA) but in the next decade, particularly with the work on electric vehicles, these numbers will only get better. The current SOA for normal electrolytics are up to $0.3 \mathrm{mWh} / \mathrm{g}$ $1 \mathrm{~J} / \mathrm{g}$, supercapacitors are up to $15 \mathrm{mWh} / \mathrm{g} \sim 54 \mathrm{~J} / \mathrm{g}$, NiFe batteries $30 \mathrm{mWh} / \mathrm{g} \sim 100 \mathrm{~J} / \mathrm{g}$ with some graphene enhanced units at $100 \mathrm{mWh} / \mathrm{g} \sim 330 \mathrm{~J} / \mathrm{g}$, NiMH batteries $60 \mathrm{mWh} / \mathrm{g} \sim 200 \mathrm{~J} / \mathrm{g}$, Ni-H2 batteries $75 \mathrm{mWh} / \mathrm{g} \sim 270 \mathrm{~J} / \mathrm{g}, \mathrm{Li}-$ ion batteries $250 \mathrm{mWh} / \mathrm{g} \sim 900 \mathrm{~J} / \mathrm{g}$. The operating temperature is also a major issue since batteries and capacitors usually have electrolytes that would freeze out at low temperatures. If needed we would use the RTG to keep them warm.

The issue is the lifetime of both the capacitors and batteries for an extremely long mission life is an issue. Here there is a lot of work from terrestrial and space systems. Both terrestrial (Ni-Fe, NiMH) and space batteries (Ni-H2) have shown extremely long lifetimes for modest depth of discharge (DOD). For example Ni-H2 space based cells have lasted approximately 20 years with 20,000 cycles at $70 \%$ DOD and $>40,000$ cycles at $40 \%$ DOD. It is unclear what the ultimate lifetime of these cells are. NiMH cells for terrestrial use in EV's have exceeded 20 years and more than 300,000 cycles (at less than 30\% DOD) in literally a million vehicles (Prius for example). Ni-Fe batteries, first developed 
more than 100 years ago (Edison cell) have shown more than 50 year life. Li cells are in rapid development and they may also meet our lifetime needs with improved development.

The area of energy appears to already meet our needs for the toughest case (wafer scale) though miniature cells and high TRL still need to be proven. Again, thermal issues need to be considered and the RTG power can be used to keep the batteries/capacitors warm.

If we desire to burst a 1 watt laser comm for $1 \mathrm{sec}$ with an electrical to photon efficiency of $76 \%$ we need an energy storage of about $1.5 \mathrm{~J}$. Derating this by a factor of 4 so we have a 25\% DOD to get high cycle life requires a $6 \mathrm{~J}$ energy storage. With all of the electrochemical cells and many of the super capacitors this requires less than $0.1 \mathrm{~g}$ of mass which is within our mass margin for even the wafer scale case.

\section{Chip level fabrication of super capacitors}

Recently (2013), the Leibniz Institute for Solid State and Materials Research Dresden developed completely monolithic all solid-state, micro-supercapacitors with high performance based on $\mathrm{MnO}_{\mathrm{x}} / \mathrm{Au}$ multilayers. With energy density of $1.75 \mathrm{mWh} / \mathrm{cm}^{3}$ and a maximum power density of 3.44 $\mathrm{W} / \mathrm{cm}^{3}$. After 15000 full discharge cycles it was still $74 \%$ of its original capacity. This is a good start and though not as high an energy density as non-chip level super caps. Additionally, microsupercapitor technology is an area of active ongoing research due to high demand for microelectronics applications, and improved technology (i.e., carbon nanotubes) will allow us to use burst power more frequently with less degradation.

It is also conceivable that continuous transmission at a received rate of one bit per second could be achieved without the need to use bursts for communication. This would enable us to use more bursts to take measurements with the magnetometer, which is the most power-hungry of the instruments we are considering. We can either reduce the magnetometer requirements for ISM studies in order to reduce the power or fold in improvements in the SOA of the magnetometers as further progress is made in reducing the power of ultra-sensitive magnetometers. The camera and spectrometer have low enough power consumption that they could be operated continuously. The bottom line for energy storage is that, while work remains to be done to test very long lived storage, the current SOA is already at the level of our needs in terms of energy storage density for the worst case of the wafer scale system.

\section{Power Generation}

We previously discussed the possibility of using radioisotope thermoelectric, piezoelectric, or betavoltaic generators onboard our probe. While all three technologies are possibly viable, we believe thermoelectrics show the greatest promise for use in the near future. Plutonium-238, the traditional fuel source for radioisotope thermoelectric generators (RTGs) produces $560 \mathrm{~mW} / \mathrm{g}$ of heat in its pure form, and $390 \mathrm{~mW} / \mathrm{g}$ of heat in its fuel pellet form (Plutonium Dioxide). Current RTG technology (NASA's Multi-Mission RTG, or MMRTG for short) has an electrical conversion efficiency of 6-7\%. Assuming $6.5 \%$ efficiency from thermal to electrical conversion we get about $25 \mathrm{mw}$ (electrical)/g. In order to generate $5 \mathrm{mw}$ we need about $0.2 \mathrm{~g}$. Since the RTG is by nature decaying we need to size the systm so at the end of mission (EOM) we get $5 \mathrm{mw}$. Pu-238 has a half-life of 87.74 years. This means that it decays at a rate of:

$$
\left(1-0.5^{\frac{1}{87.74}}\right)=.00787 / \text { year }
$$


RTGs also experience thermocouple degradation ( .008 per year for the MMRTG) [11]. Therefore we can expect a loss of power output of about $1.6 \%$ per year. Assuming a 20 year mission we can calculate the amount of initial $\mathrm{Pu}-238$ dioxide needed.

$0.2 \mathrm{~g}=$ Required initial amount of $\mathrm{Pu}-238$ dioxide $\mathrm{e}^{-0.016 / \text { year-20years }}$

$\Rightarrow$ Required initial $(\mathrm{BOM})$ amount of $\mathrm{Pu}-238$ dioxide $=0.275 \mathrm{~g}$

We will generate about $38 \%$ more power at the BOM than the EOM.

Since the unit is only assumed to be $6.5 \%$ efficient and most of this also ends up as heat we have a total heat supply of about $5 \mathrm{mw}$ x 100/6.5 77mw. This can be used to heat the wafer or elements of it if needed. Depending on the final system architecture we may want to use this heat during the long cruise phase.

The density of $\mathrm{Pu}-238$ dioxide fuel pellets is $9.6 \mathrm{~g} / \mathrm{cm}^{3}$.

$\left(\frac{.275 \mathrm{~g}}{9.6 \mathrm{~g} / \mathrm{cm}^{3}}\right)=0.0286 \mathrm{~cm}^{3}=28.6 \mathrm{~mm}^{3}$ or about a $3 \mathrm{~mm}$ cube. This easily on a small section of our wafer. The technology development needed here is to miniaturize the RTG packaging. Similar power RTG's were used in early pacemakers and thus there is already precedent. These were obviously not space qualified so the TRL level is low. This is on the technology development roadmap.

\section{Options for MEMS and miniature Stirling engines}

RTG's using thermoelectric converters are only about 6-7\% efficient currently. We will do slightly better as we have a colder heat dump but still this is a low efficiency system. Stirling engines are much more efficient with about 30-50\% efficiency for the temperatures we can get BUT there are no chip scale Stirling engines and no 20-100 year lifetime Stirling engines currently exist even without the extreme requirement of chip level system. MEMS equivalents may be possible but this is a research item yet to be explored. This leaves open the possibility of much more power (by a factor of 5-10) that may be achieved. This would greatly expand our data rates as well as sensor suite possibility for the small systems. For the larger mass spacecraft systems this is much less of an issue. This is another area for exploration and possible TRL maturation.

\section{Scalability of Wafer Scale Thermoelectrics with RTG's}

Although thermoelectric generators present some challenges due to difficulty in maintaining temperature differences at small scales, recent work with silicon nanowires for thermoelectric conversion at UCSB has demonstrated a power generation of $29 \mu \mathrm{W}$ with a temperature difference of only $56 \mathrm{~K}$ in a $50 \mu \mathrm{m} \times 50 \mu \mathrm{m}$ device. This is ideal for a wafer level RTG converter as the Si nanowires can made on the wafer. We will have much more than a $56 \mathrm{~K}$ temperature difference and thus would expect much higher power production for a similar device. We assume a worst case scenario and use the existing device with a $56 \mathrm{~K}$ temperature difference. In order to generate $5 \mathrm{mw}$ we would need to scale up the area by a factor of $5000 \mu \mathrm{W} / 29 \mu \mathrm{W} \sim 170$. Thus we would need an area 170 time larger or about $650 \times 650 \mu \mathrm{m}$. This is a small fraction of the wafer.

Small RTGs for 20-year missions have been developed based on the Light Weight Radioisotope Heater Unit which is $63.5 \mathrm{~mm}$ x $130 \mathrm{~mm}$, 'weighs' $315 \mathrm{~g}$, and outputs $1 \mathrm{~W}$ of heat. Additionally, the U.S. Navy "superbattery" was a $500 \mathrm{~mW}$ RTG developed in the 1970's, which was a cylinder 1.6 in x 4 in. It was reported that reduction in size would be possible by redesigning the heat source. In the 1970 's, even smaller RTGs were used to power pacemakers. One that generated $0.3 \mathrm{~mW}$ was 
developed by Numec Corporation under contract from the U.S. Atomic Energy Commission. Thus there are numerous precedents for very small RTGs and some chip level work at UCSB makes wafer scale systems feasible.

\section{Alternative Possibilities for the RTG}

Other possible choices of isotope include Americium-241, Strontium-90, Tritium, and Nickel-63. These have all been eliminated in favor of $\mathrm{Pu}-238$ because they possess smaller energy densities and/or shorter half-lives. Betavoltaics and piezoelectrics are another option we have looked at. The Betacel 400 (another 1970's technology) was a betavoltaic nuclear battery powered by Promethium-147 that was used in pacemakers and generated $0.37 \mathrm{~mW}$. Betvoltaics are a backup option because modern incarnations (such as the NanoTritium nuclear battery produced by City Labs) tend to have lower power densities than RTGs (mainly because the beta-emitters they use, such as Ni-63 or Tritium, are not as power-dense as $\mathrm{Pu}-238$ ). Cornell University developed a Ni-63 piezoelectric generator using $2.9 \mathrm{mCi}$ of Ni-63 that was $\sim 4 \%$ efficient. The same group of researchers is involved with a DARPA project to create power supplies for Hybrid Insect Micro-Electro-Mechanical Systems (HI-MEMS), which are essentially cybernetic insects, and accordingly require very small power supplies. Again, the disadvantage is the power density of Ni-63 compared to $\mathrm{Pu}-238$. It could, however, be a viable backup option.

\section{Using the directed energy beam itself to power the spacecraft}

One option is to use the drive laser as a source of power. While the beam spreads with increasing distance of the spacecraft this can still be quite useful, particularly in the earlier phases of the mission. One option here is to develop narrow bandgap PV junctions that are tuned to the laser. This needs to take into account the Doppler shift of the laser. In the cases the Doppler shift is very substantial ( $25 \%$ ) and hence this needs to be designed in. The reason to use narrow bandgap PV is to increase the efficiency compared to wide bandgap PV as is commonly used for terrestrial PV. With space PV at $50 \%$ for concentrated wideband PV we may be able to do even better for narrow bandgap PV. Another options that combines the laser PV conversion for the initial part of the journey with the wideband PV needed upon arrival (see below) is to make hydrid junctions with both multi junction broadband PV with one additional narrow band junction for the laser. That way one PV works for both cases. No such cell has even been built but discussions with PV cell and materials experts have convinced us it is feasible to do and would likely yield a very efficient laser PV converter.

\section{Using photovoltaics upon arrival to increase power}

Another option is to use PV upon arrival at a stellar system. This can be particularly useful in passes that are close enough to have Earth like illumination. At the earth we have about $1400 \mathrm{~W} / \mathrm{m}^{2}$ or 140 $\mathrm{mW} / \mathrm{cm}^{2}$. Current space PV is nearing $40 \%$ efficiency in non concentrated form and already at $50 \%$ for concentrated. If we assume $50 \%$ conversion we get an electrical power of $70 \mathrm{mw} / \mathrm{cm}^{2}$. The hardest case (as usual) is the wafer scale case. There are several ways to envision using this option. One is to imagine the sail itself focuses the starlight ("sunlight") or is covered with PV. The former is lower mass in general. In either case you get about the same power. The other option is to simply cover a portion of the wafer with PV or use the backside of the wafer as all PV. With the $1 \mathrm{~m}$ class sail the power available is about 500 watts and with the wafer alone the power available is about 7 watts. These are clearly enormous numbers compared to the RTG. This opens up a dramatic possibility of radically increasing the data rate back to earth upon arrival among other possibilities. Other 
possibilities include increase maneuvering and increased braking to execute orbital opportunities. A detailed orbital dynamics simulation needs to be done to further explore the maneuvering possibilities.

\section{Hydrid PV for both laser and stellar arrival}

Another options that combines the laser PV conversion for the initial part of the journey with the wideband PV needed upon arrival is to make hydrid junctions with both multi junction broadband PV with one additional narrow band junction for the laser. That way one PV works for both cases. No such cell has ever been built but discussions with PV cell and materials experts have convinced us it is feasible to do and would likely yield a very efficient laser PV converter.

\section{Attitude Control}

We propose photon-thruster attitude control, with at least one such thruster mounted on each opposite edge of the wafer, firing tangentially to the wafer to create a torque and cause rotation. We will show that such a system is feasible.

First, from a pure energetics perspective:

Rotational Kinetic Energy $=\left(\frac{1}{2}\right) \mathrm{I}^{2} \mathrm{I}_{\text {rectangular plate }}=\left(\frac{1}{12}\right) \mathrm{ML}^{2}$

Where $\mathrm{L}$ is the length of the rotating side of the plate [29]. Therefore $1 \mathrm{~J}$ of rotational KE will result in the wafer rotating by:

$\omega=\left(\frac{24}{\mathrm{ML}^{2}}\right)^{\frac{1}{2}}=\left(\frac{24}{0.001 \cdot 0.1^{2}}\right)^{\frac{1}{2}}=1549 \mathrm{radians} / \mathrm{s}=246 \mathrm{rev} / \mathrm{s}$

Similarly, $1 \mathrm{~mJ}$ of rotational KE amounts to $7.8 \mathrm{rev} / \mathrm{sec}, 100 \mu \mathrm{J}$ to about $2.5 \mathrm{rev} / \mathrm{sec}, 10 \mu \mathrm{J}$ to .78 $\mathrm{rev} / \mathrm{sec}$, and $1 \mu \mathrm{J}$ to about $.25 \mathrm{rev} / \mathrm{sec}$. Therefore, if we are able to impart just $1 \boldsymbol{\mu J}$ of rotational KE to our wafer, it will result in rotation at a rate of (very nearly) $90 \%$ second. Unfortunately, imparting even a $\mu \mathrm{J}$ of rotational $\mathrm{KE}$ to the wafer is not feasible with photon thrusters utilizing onboard power generation. However, maneuvering the wafer still proves feasible when we calculate the thrust of such an attitude control system.

\section{Photon Thrusters}

We will use edge mounted LED's or laser diodes as photon thrusters for wafer orientation control. Photon thrust is $\mathrm{P} / \mathrm{c}$ where $\mathrm{P}$ is the photon thrust power. This is about $3.3 \mathrm{nN} / \mathrm{W}$ or $3.3 \mathrm{pN} / \mathrm{mW}$. If we assume that our photon thrusters are allotted an equal amount of power and are located on opposite edges of the wafer facing in opposite directions, this thrust is converted directly into a torque, causing the wafer to rotate.

$$
\Gamma=\mathrm{r} \cdot \mathrm{F}=0.05 \mathrm{~m} \cdot 3.33 \times 10^{-12} \mathrm{~N}=1.67 \times 10^{-13} \mathrm{Nm} \quad \Gamma=\mathrm{I} \alpha \Rightarrow \alpha=\frac{\Gamma}{\mathrm{I}}=2 \times 10^{-7} \mathrm{rad} / \mathrm{sec}^{2} / \mathrm{mW}
$$

If we temporarily dedicate all $5 \mathrm{~mW}$ of onboard power to thrust production, we achieve

$$
\begin{aligned}
& \alpha^{\prime}=.76 \cdot 5 \mathrm{~mW} \cdot 2 \times 10^{-7} \mathrm{rad} / \mathrm{sec}^{2} / \mathrm{mW}=7.6 \times 10^{-7} \mathrm{rad} / \mathrm{sec}^{2} \\
& \theta=\omega \mathrm{t}=\int_{0}^{\mathrm{t}} \alpha^{\prime} \mathrm{t}^{\prime} \mathrm{dt}^{\prime}=\frac{1}{2} \alpha^{\prime} \mathrm{t}^{2} \quad \theta=\frac{\pi}{4} \Rightarrow \mathrm{t}=\sqrt{\frac{\pi}{2 \alpha^{\prime}}}=1,438 \mathrm{sec}=24 \mathrm{~min}
\end{aligned}
$$


Where $\alpha$ is the angular acceleration using $1 \mathrm{~mW}$ to power the thrusters, $\alpha^{\prime}$ is the angular acceleration using $5 \mathrm{~mW}$ at $76 \%$ (electrical to optical - currently achieved) efficiency, and $\theta$ is the angle through which the wafer has rotated.

This calculation tells us that it will take 24 minutes for the wafer to rotate through a $45^{\circ}$ angle. It will have nonzero angular velocity $\left(\sim 5.46 \times 10^{-4} \mathrm{rad} / \mathrm{sec}\right)$, which we will cancel by immediately firing our thrusters in the opposite direction. Thus after 48 minutes have passed (decelerating the wafer will require exactly the same time as accelerating, and $2 \times 24=48$ ), our wafer will have achieved a $90^{\circ}$ rotation about its axis and it will have zero angular velocity when it reaches that position. This result is both surprising and fortuitous, and reveals yet another advantage of the wafer-scale probe: low-energy maneuverability.

\section{Wafer Substrate}

We have selected Gallium Arsenide as our material of choice for the wafer substrate, with Silicon

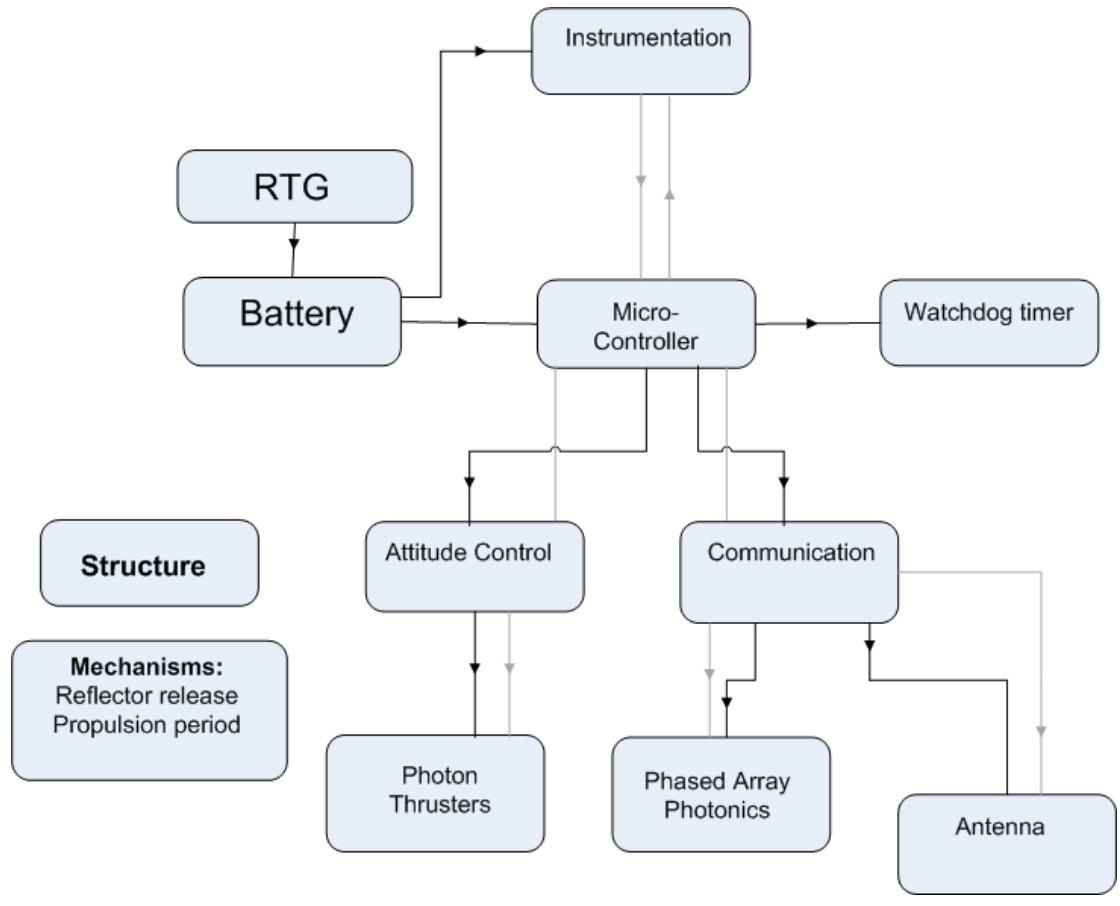

Figure 5. Box-Arrow Diagram of Electronics on-board.
Germanium and InP as our backup option.

One of the main advantages of GaAs is its greater electron mobility, due to the reduced effective mass of its charge carriers (assuming equivalent doping). This allows for higher electron velocities, resulting in higher operation frequencies and improved logic switching speeds. The capability for higher frequencies reduces spectrum crowding and GaAs advances have also resulted in lower power consumption, which is important for our purposes. GaAs is also highly resistive, which increases its natural insulation properties, thereby reducing 'cross-talk' between devices. This allows the integration of radio-frequency devices, logic devices, transmission lines, and passive elements on a single substrate - again, important for a wafer probe. GaAs integrated circuits are lighter, higher-performance, and suffer from fewer parasitics in discrete device packaging than ICs made from other materials.

GaAs also produces less noise than other semiconductors, and is therefore superior for long-range, low-power communications. It is less sensitive to heat than $\mathrm{SiGe}$, and has greater radiation resistance. As a result of all these properties, GaAs is already commonly used in space applications, and is the most suitable material for our particular application as well.

\section{System Architecture}


Here we will treat the case of the $1 \mathrm{~g}$ wafer-scale spacecraft, because it represents the most difficult engineering feat. If we can successfully design a Wafer probe at the wafer level, the scalability of our architecture ensures that a larger craft could also be constructed. As the reverse is not true, the architecture of the wafer-scale probe is considered to be the most essential to the ultimate success of our design. The wafer architecture is centered on the concept of the integrated circuit (IC). An IC is a circuit wherein all of the components are crafted out of the same block of semiconductor material, known as a monolith. The main problem that ICs were developed to solve was that as circuits became smaller and smaller, they required more and more wiring in less and less space, without sacrificing the integrity of the connections. ICs solved this problem by doing away with conventional wires entirely, replacing them with a thin layer of metal printed in the shape of the necessary connections directly onto the monolith which contained all of the circuit components. Our wafer will run cold during the majority of the mission (around 20-50K) and this will lead to even higher performance (lower leakage currents) especially suitable for GaAs or $\mathrm{SiGe}$ or $\mathrm{InP}$ based semiconductors we baseline. Normal $\mathrm{Si}$ is generally not suitable at these temperature though some special doping Si devices have been used in IR arrays for military and space related purposes.

\section{Major breakthroughs}

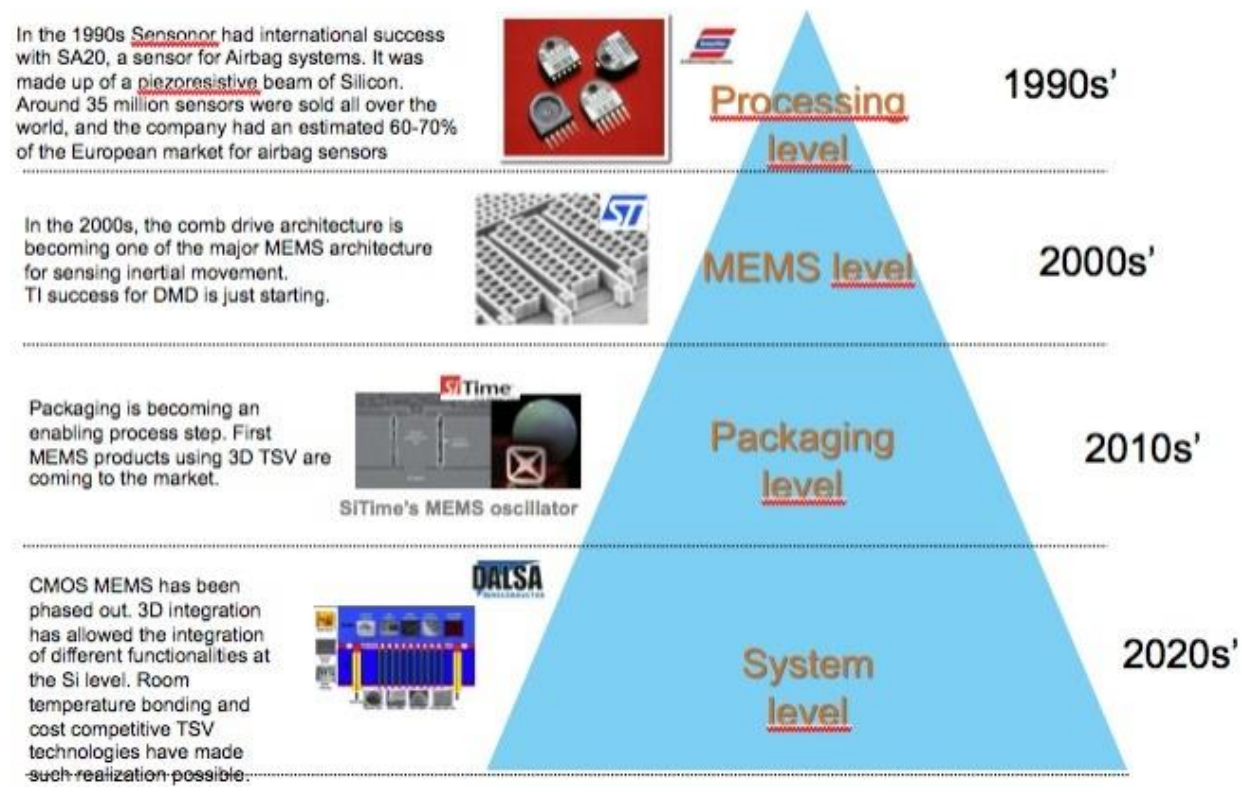

Figure 6. Diagram of recent and futuristic breakthroughs in Wafer technology.
One of the great advantages of ICs is that once the masks have been created, they can be used over and over, facilitating low-cost mass production. This is a key element of our "shotgun approach" to sending a large number of wafer-scale probes. They can be produced en masse at low cost with modern IC wafer printing techniques. Current state-of-the-art wafer IC technology allows for singlefunctionality systems to be printed (i.e., wafer-

level cameras), with multi-function wafer ICs (i.e., wafer-level spacecraft) on the horizon. Research companies such as Fraunhofer are actively working on "heterogeneous integration of different components, such as sensors, processors, memories or antennas". This is precisely what will be necessary in order to build a wafer-level spacecraft. It has been nearly 60 years since the advent of the integrated circuit, and the technology continues to develop at an incredible rate. Wafer-level MEMS technology is steadily improving as well, which bodes well for the future of wafer IC technology (and printed spacecraft). 

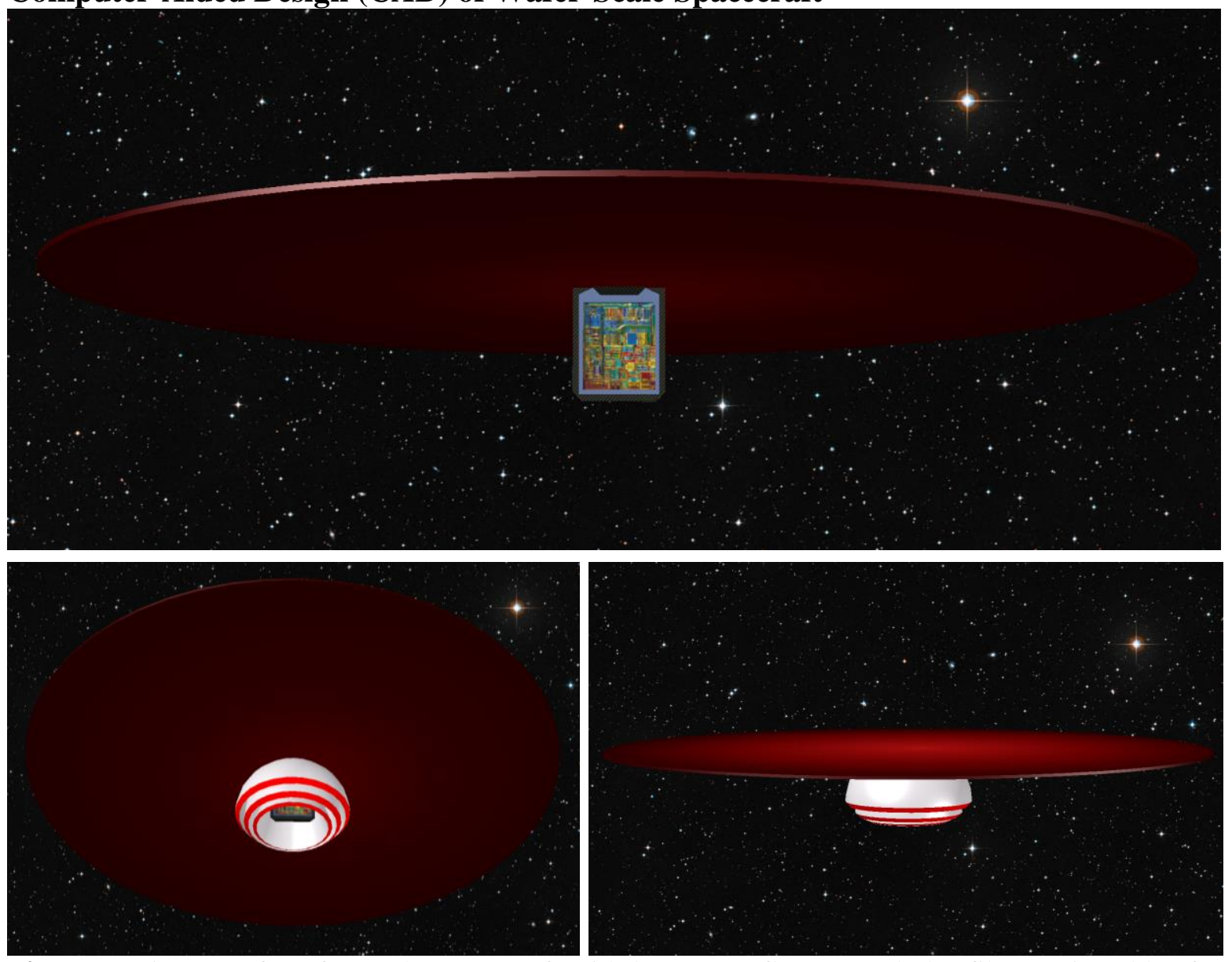

Figure 7. Pictures of Wafer Scale Spacecraft with laser on reflector. Includes fiber optic cables for cloaking and wafer as the payload. The red depicts the laser light.

\section{Reflector Stability and Shaping}

A critical issue will be the stability of the sail. There are a number of perturbative effects. These include laser instabilities and laser mode issues, differential forces on the sail and mechanical modes in the sail, heating of the sail and laser pointing instability.

This is a complex sets of issues that requires a significant amount of research and development. This will not be trivial. Some ways to mitigate these issues are spinning the sail (especially if circular) and shaping of the reflector into a slight conic (similar to a reentry vehicle). Feedback from the sail to the laser will help but the time of flight will lower the effective servo bandwidth for this. Ideally a self stabilizing system is desired. We see this as one of the most critical issues to overcome.

\section{Reflector Structure}

The issue here is to keep the reflector in the proper shape particularly during the acceleration phase and to do so with minimal mass. One idea is to use centrifugal force to keep the sail tight through tension. We would spin our spacecraft to a sufficient rotational speed, enough so that the added tension on the sail due to the centrifugal force exerted on it would provide sufficient structural support for the sail to not collapse. As an analogy, imagine a drum. We propose a design, in which our sail would be 
stretched over a circular ring of a strong, rigid material, just like the skin of a drum is stretched over its rim. This would then be spun before launch. This combination of structure and rotational speed might be a potential solution.

Our second idea completely diverges from the first. In this concept we envision using a support structure that consists of an inflated shell/bubble. This inflated shell would be behind the reflector. The pressure on the sail caused by the laser, would cause an increase in the pressure in this inflated structure. This increased pressure would counteract the pressure on the sail. Thus, there would be a sufficiently strong enough counteracting force so that our sail would not collapse under the pressure of the laser.

These are starting points that will lead to more concise, plausible and feasible designs.

\section{G-Forces on Small-Scale Spacecrafts}

Possibly the greatest benefit of our wafer scale design is the high speeds our spacecraft can reach. We have discussed the ability of our ship to be accelerated to about $0.25 \mathrm{c}$ in ten minutes. This is an acceleration of roughly 10,000 g's, an acceleration that could put a formidable strain on our delicate wafer. However, this may not be as big of a problem as it may seem.

Many present day weapons systems incorporate electronic components into their artillery shells to correct trajectory mid flight. During launch, these electronic components must be able to sustain accelerations of at least 10,000 g's, sometimes ranging to even higher than 15,000 g's depending on the system. Upon muzzle exit, the artillery shells are subject to substantial pressure changes, resulting in significant shocks and vibrations [12]. Our spacecraft would not be subject to such volatile environment, as our acceleration takes place over a period of at least ten minutes, rather than a fraction of a second. Many methods have been successfully developed to house electronic components during launch, most involving some sort of shock absorbing material such as foam or gel [13]. It is reasonable to think that a similar method could be used for our spacecraft.

\section{Photon recycling for larger thrust and efficiency}

The efficiency of the photon drive can be improved by reusing the photons reflected by the spacecraft reflector in an effective optical cavity mode to get multiple photon reflections. This is known as photon recycling. It is not a new concept but may be of some use for some of our applications. We will see it greatly complicates the system optics however. To understand this we need to first discuss the efficiency of the conversion of the laser photons into motion of the spacecraft.

\section{Energy and Momentum Propulsion Efficiency}

The instanteous energy efficiency (power that goes into direct kinetic energy/ laser power on reflector) $\varepsilon_{\mathrm{p}}=\beta\left(1+\varepsilon_{\mathrm{r}}\right)=\mathrm{P}_{\mathrm{o}} \mathrm{t}\left(1+\varepsilon_{\mathrm{r}}\right)^{2} / \mathrm{mc}^{2} \sim 2 \beta \sim 4 \mathrm{P}_{\mathrm{o}} \mathrm{t} / \mathrm{mc}^{2}$ for $\varepsilon_{\mathrm{r}} \sim 1$ and total integrated energy efficiency $\varepsilon_{\mathrm{total}}=$ $1 / 2 \varepsilon_{\mathrm{p}}=\beta\left(1+\varepsilon_{\mathrm{r}}\right) / 2=\mathrm{P}_{\mathrm{o}} \mathrm{t}\left(1+\varepsilon_{\mathrm{r}}\right)^{2} / 2 \mathrm{mc}^{2} \sim \beta \sim \mathrm{P}_{\mathrm{o}} \mathrm{t} / \mathrm{mc}^{2}$ for $\varepsilon_{\mathrm{r}} \sim 1$ where $\mathrm{m}=\mathrm{m}_{\text {sail }}+\mathrm{m}_{\mathrm{o}}$, momentum "eff" $=\left(1+\varepsilon_{\mathrm{r}}\right) \sim 2$ for $\varepsilon_{\mathrm{r}} \sim 1$ with $\beta<<1$.

The energy transfer efficiency starts out at very low levels and then increases proportional to the speed. The total integrated energy efficiency is just $1 / 2$ that of the instantaneous efficiency at the final speed since the force is constant as long as the laser spot is smaller than or equal to the reflector size and hence the acceleration is constant and hence speed increases proportional to time $(\beta \sim \mathrm{t})$ and hence the average $\beta$ is $1 / 2$ the maximum $\beta$ achieved. This is for the non relativistic case. For spacecraft accelerated to high speeds the energy efficiency can become quite high and approaches unity. 
In the case of photon recycling the photons bounce back and forth in an optical cavity one end of which is the spacecraft reflector and the other end is a relatively more massive (referred to here as fixed) mirror. The total power at the spacecraft mirror sets the force on the spacecraft. The total power on spacecraft mirror is essentially the same as that on the fixed mirror. The combination of the two mirrors forms an optical cavity whose $Q$ factor is defined as $Q=2 \pi E_{\text {cav }} / E_{\text {loss }}$ where $E_{\text {cav }}=$ Energy stored in cavity and $\mathrm{E}_{\text {loss }}=$ energy lost per cycle. One cycle is the round trip travel time of the light or $2 \mathrm{~L} / \mathrm{c}$ where $\mathrm{L}$ is the distance between the spacecraft and fixed mirror. In general the fixed mirror will be at the laser driver (ie near the earth). The energy lost per cycle is due to a variety of effects such as increase of kinetic energy of the spacecraft and fixed mirror per cycle, energy lost to mirror(s) absorption per cycle due to non unity reflection coefficient, diffraction effects as the spacecraft moves away and mirror misalignments. For the spacecraft close to the laser optical cavities are possible and do improve efficiency (the effective power on the spacecraft reflector increases by the number of "bounces". As the spacecraft begins to move far away diffraction becomes extremely problematic as do mirror alignment issues and hence photon recycling has much less practical use. It is an area we are exploring but it greatly increases the complexity of the system as it requires extremely large optical cavity mirrors on the Earth side.

\section{Backgrounds for Communications}

The relevant backgrounds at $1 \mu \mathrm{m}$ wavelengths are optical emission from the telescope/ array, zodiacal emission from our solar system dust both scattering sunlight and emitting thermal radiation (Zodi).

The Zodiacal light is highly anisotropic and also time dependent and location of the Earth in the orbit around the sun dependent. We treat this from data collected from the DIRBE instrument on COBE. The CIB is far more isotropic on modest angular scales and becomes largely point like on very small scales. Again we model this from the DIRBE data on COBE and subsequent measurements. We also model the optics at various temperatures and the Earths atmospheric emission for inside the atmosphere measurement but will focus here on orbital programs. Please see Riley etal 2013 [7].

\section{Relativistic Dust}

Interstellar dust poses a significant threat to the survival of a 1 gram spacecraft traveling at quasirelativistic speed. While some interesting means of protection have been proposed for larger missions, such as the innovative "shield cloud" [14], these methods are likely not feasible when the total payload mass is limited to 1 gram (Daedalus, for instance, concluded that beryllium armor, which represented the upper limit of mass loss due to erosion, yielded a loss of armor about $9 \mathrm{~mm}$ thick. At a density of $1.85 \mathrm{~g} / \mathrm{cm}^{3}$, even a $10 \mathrm{~cm}$ x $9 \mathrm{~mm}$ x $1 \mathrm{~mm}$ shield would weigh 1.665 grams. While a boron shield would experience less erosion, it is also denser) [15].

The shield cloud Daedalus proposed a cloud $100 \mathrm{~m}$ thick with a mass of $6 \mathrm{~kg}$ as an effective means of protection against dust particles at speeds up to $0.12 c$ [14]. This was intended to protect a frontal area of $3217 \mathrm{~m}^{2}$ [50]. As the frontal area of our probe is only about $.005 \mathrm{~m}^{2}$, this would reduce the crosssectional area of the required shield cloud by a factor of over one million, leading to a cloud mass of about $10 \mathrm{mg}$ (this, however, assumes the same $100 \mathrm{~m}$ cloud thickness, which would, of course, need to be increased to account for our higher speed. However, even if we required a cloud 10 times as thick, its required mass would still be only .1g). The more significant problem here is not the mass of the shield could itself, but rather the deployment mechanism. Daedalus proposed the use of selfpropelled "bugs" which would fly ahead of the spacecraft and then release the dust clouds [14]. The 
design of a similar deployment mechanism at the required scale represents a significant engineering challenge; one which we shall not assume will be met within the required time frame.

For these reasons, we propose the "shotgun approach." Because our probe accelerates to .26c in only 10 minutes, and is scaled to be affordably produced (wafer-printed with mass-production capabilities in mind), it would be feasible to send a large number of probes in a relatively short period of time, and expect that some of them will be destroyed. In fact, the discovery of the percentage which survive (and how far they travel before a destructive collision) will be a relevant scientific return of this mission, as it will improve our understanding of the distribution of interstellar dust. This will in turn contribute to the development of enabling technologies for future missions.

\section{Mag Sail for Deceleration}

For our larger spacecraft we might be able to implement a magnetic sail in order to slow the probe down as it approaches its target destination. Magnetic sails work by passing a current through a loop of superconducting cable, thereby ionizing nearby interstellar particles and subsequently deflecting them. This deflection requires a transfer of momentum from the probe to the particles it deflects, thereby slowing it down. Furthermore, futuristic superconducting materials, which may be available by 2040 , would greatly increase the effectiveness of the magnetic sail. A magsail utilizing an YBCO superconductor $(\mathrm{Tc} \sim 100 \mathrm{~K})$ could feasibly decelerate a relativistic probe from interstellar cruising speed to interplanetary speeds in less than one year. [16] This would represent the ideal case for our mission since we are running at temperatures less than $100 \mathrm{~K}$ and thus we can use high Tc superconductors. This also opens up possibilities for high Tc elements on our wafer. We could also pass very close to the star in the target system (within $0.6 \mathrm{AU}$ ) to take advantage of the solar wind and the greater particle density in the astrosphere to increase deceleration. [17] This is an area for future research to determine if this is truly compatible with our systems. Our baseline does not assume orbiting capability but this would clearly be a preferable option and one we are exploring further. The practicality of a magsail for slowing and orbiting a star is not been properly analyzed. Other options

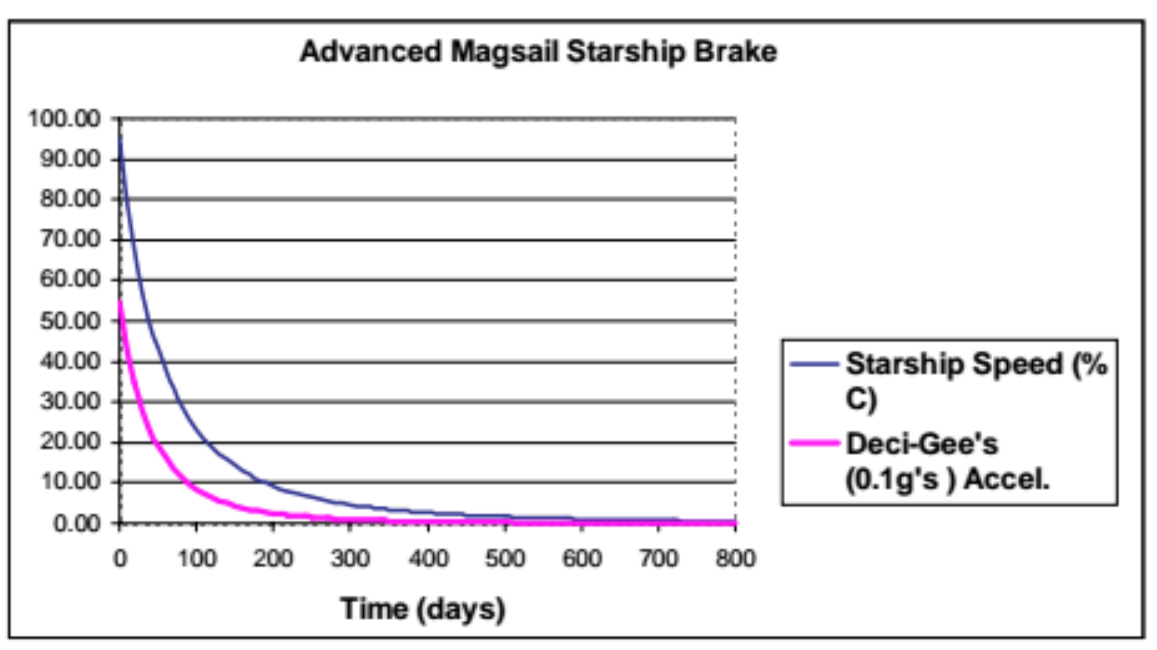

Figure 8. Advanced Magsail deceleration using YBCO Superconductor for a $100 \mathrm{~km}$ radius magsail. Magnetic Field of $\mathrm{Bm}=1.0 \mathrm{x} 10-6 \mathrm{~T}$, with a wire density of 7 X $103 \mathrm{~kg} / \mathrm{m} 3$, an engineering current density of $1011 \mathrm{~A} / \mathrm{m} 2$ at $77 \mathrm{~K}$, a 1.53 $\mathrm{mm}$ thick wire, and a payload ratio of 0.8088 . to be explored include the new esail (electric sail) discussions (solar wind driven) and photon braking, but again, neither has been properly simulated for a real flight scenario. One first analysis of photon braking using the light from the star to brake by passing very close to the host star does not look feasible for decreasing speed to orbital speeds needed to enter a planetary orbit $(\sim 10 \mathrm{~km} / \mathrm{s})$. This needs much more study to raise the TRL. 


\section{Economic Feasibility}

In addition to the use of the laser driver for interstellar flight it can be used for the many other uses our group has published; not the least of which is virtually complete planetary defense as well as using the system for space to Earth power which again amortizes the investment. The cost of the spacecraft would be much smaller than the cost of the laser driver and with the wafer scale technology we propose mass production would be enabled thus amortizing the NRE costs. Still realistic economic planning is needed to make this program feasible which is why we propose staged development with realistic milestones.

As for the cost of the spacecraft, the WaferScale designs allow for leveraging of the semiconductor industry and capitalize on the rapid advancements in this area. The NRE costs of a wafer design are significant (10-100M\$) but can be amortized over the mass production of wafers with the costs for even GaAs or SiGe being quite reasonable. The development costs for the spacecraft reflectors will be significant if we want to get to 1 micron thick reflectors though there are several micron thick plastic film reflectors for solar sails but they are not suitable for high flux laser use. Currently micron level glass sheets exist that should allow us to achieve good dielectric coated reflectors.

\section{Science Objectives and Instrumentation}

For an initial mission, we propose outfitting our probe with a microspectrometer, a micromagnetometer, and a MEMS camera. With a spectrometer aboard, we could search for recent or nearby sources of cosmic ray acceleration, determine the relative fraction of cosmic ray electrons and positrons in the interstellar medium, and search for antiprotons produced by Hawking radiation or the decay of the Weakly Interacting Massive Particles (WIMPs) which may account for the missing dark matter. A magnetometer would allow us to measure suprathermal ions and electrons in the solar wind, help characterize the structure of the bow and termination shocks, determine the nature of flows in the heliosheath, discover why the magnetic flux dropped at Voyager 1, help determine why the heliosheath is almost 50\% thinner than models indicate it should be, determine the relationship between solar and interstellar magnetic fields, measure the interchange of solar and interstellar ions, determine the nature of instabilities in the heliopause, and the nature of interstellar magnetic fields. And finally, a camera allows us to observe extragalactic background light, zodiacal and Kuiper Belt dust distributions, and zodiacal background. More importantly, however, the camera helps us to feel like we are actually out there, and generates and maintains public interest by providing images of the universe beyond our solar system. [18]

For future missions, some or all of these instruments could be exchanged for others which would allow different tests and discoveries, including, but not limited to, atomic clocks, accelerometers, plasma detectors, dust composition instruments, and small ( $\sim 3 \mathrm{~cm}$ class) telescopes. With these we could test the Equivalence Principle, fundamental constants, local position invariance, the gravitational inverse square law at large distances, the one-way speed of light, search for gravity waves, measure the Edington parameter to a greater precision than ever before, and measure the parallax of distant objects, as well as take plasma measurements of the solar wind, heliopause, heliosheath, and bow and termination shocks. [18]

\section{Payload Options and Parameters}


Once a suitable laser driver is built the payloads can be any size from miniature relativistic probes, such as the wafer scale one for interstellar flight we have discussed, to large spacecraft capable of transporting humans in the solar system. Some examples of the many mission scenarios possible are shown below. Note the single laser driver can be used launch sequentially or in parallel any number of spacecraft and thus the system enables and is amortized over a large mission space. The following gives a selected set of possible missions. It is assumed that the reflector mass is equal to the base spacecraft mass (i.e. system mass not including reflector - or total system mass is twice the base spacecraft mass). The reflector is assumed to be 1 micron thick and the reflector density is assumed to be $1.4 \mathrm{~g} / \mathrm{cc}$. The laser array is assumed to be $10 \mathrm{~km}$ on a side and the reflector is assumed to be square. The mass given is the base spacecraft mass and hence the reflector mass. The total mission mass is twice the bare spacecraft mass for the optimum speed case (Lubin 2015 JBIS). [2] [4] The laser power is assumed to be $100 \mathrm{GW}$.

\begin{tabular}{|c|c|c|c|c|c|c|c|c|}
\hline $\begin{array}{c}\text { Bare } \\
\text { Craft } \\
\text { Mass } \\
(\mathrm{kg})\end{array}$ & $\begin{array}{r}\mathrm{R} \\
\text { eflect } \\
\text { or } \\
\text { size } \\
(\mathrm{m})\end{array}$ & $\begin{array}{l}\text { Time to } \\
\text { when laser } \\
\text { diffraction } \\
\text { spot equals } \\
\text { reflector } \\
\text { size (s) }\end{array}$ & $\begin{array}{l}\text { Distance } \\
\text { when laser } \\
\text { diffraction } \\
\text { spot equals } \\
\text { reflector } \\
\text { size }(m)\end{array}$ & $\begin{array}{c}\text { Beta } \\
\text { when } \\
\text { laser } \\
\text { diffracti } \\
\text { on spot } \\
\text { equals } \\
\text { reflector } \\
\text { size }\end{array}$ & $\begin{array}{c}\text { Beta with } \\
\text { continue } \\
\mathrm{d} \\
\text { illuminat } \\
\text { ion }\end{array}$ & $\begin{array}{l}\text { Acceleratio } \\
\mathrm{n} \text { when } \\
\text { reflector is } \\
\text { fully } \\
\text { illuminated } \\
\text { (g) }\end{array}$ & $\begin{array}{c}\text { Time } \\
\text { to AC } \\
\text { system } \\
\text { (yrs) }\end{array}$ & $\begin{array}{c}\text { Reach } \\
\text { Alpha } \\
\text { Centauri } \\
\text { in } 100 \mathrm{yrs} \\
(\mathrm{y} / \mathrm{n})\end{array}$ \\
\hline 0.001 & 0.85 & 156 & $4.01 * 10^{9}$ & 0.17 & 0.24 & 33,800 & 16.6 & $\mathrm{y}$ \\
\hline 0.01 & 2.7 & 879 & $1.27 * 10^{10}$ & 0.096 & 0.14 & 3,370 & 28.5 & $\mathrm{y}$ \\
\hline 0.1 & 8.5 & 4920 & $4.01 * 10^{10}$ & 0.054 & 0.078 & 338 & 51.3 & $\mathrm{y}$ \\
\hline 1 & 27 & $2.78 * 10^{4}$ & $1.27 * 10^{11}$ & 0.031 & 0.043 & 33.7 & 93.0 & $\mathrm{y}$ \\
\hline 10 & 85 & $1.56 * 10^{5}$ & $4.01 * 10^{11}$ & 0.017 & 0.024 & 3.38 & 166.7 & $\mathrm{n}$ \\
\hline 100 & 270 & $8.79 * 10^{5}$ & $1.27 * 10^{12}$ & 0.0097 & 0.014 & 0.337 & 285.7 & $\mathrm{n}$ \\
\hline 1,000 & 850 & $4.92 * 10^{6}$ & $4.01 * 10^{12}$ & 0.0054 & 0.0077 & 0.0338 & 519.5 & $\mathrm{n}$ \\
\hline 10,000 & 2700 & $2.78 * 10^{7}$ & $1.27 * 10^{13}$ & 0.0031 & 0.0043 & 0.00337 & 930.2 & $\mathrm{n}$ \\
\hline 100,000 & 8500 & $1.56^{*} 10^{8}$ & $4.01 * 10^{13}$ & 0.0017 & 0.0024 & 0.00338 & 1,666 & $\mathrm{n}$ \\
\hline
\end{tabular}

Table 1. Reflector mass is equal to the base spacecraft mass. The reflector is square with a thickness of 1 micron and with a density of $1.4 \mathrm{~g} / \mathrm{cc}$. We assume a $100 \mathrm{GW}$ laser for these numbers $(10 \mathrm{~km})$ on a side.

\section{Power and Communication for 1g, 10g, 100g, 1kg Spacecraft}

The spacecraft listed below share the same parameters as the ones in the previous section, Payload Options and Parameters. Each spacecraft devotes $20 \%$ of the bare craft mass to an RTG for electrical 
power. For the data rates below we assume the reflector is used as the laser communication optics. This differs from the case shown in Figure 8 which only uses the $10 \mathrm{~cm}$ square wafer as the laser communication optics. The average data rate assumes continuous (100\% duty cycle) communication from Alpha Centauri so it is much lower compared to the burst data rate $(0.5 \%$ duty cycle) as shown in Table 2.

\begin{tabular}{|c|c|c|c|c|c|c|c|c|}
\hline $\begin{array}{c}\text { Bare } \\
\text { Craft } \\
\text { Mass } \\
(\mathrm{kg})\end{array}$ & Reflector & $\begin{array}{c}\text { Electrical } \\
\text { Power } \\
\text { assuming } \\
20 \% \text { bare } \\
\text { mass } \\
\text { devoted to } \\
\text { RTG(W) } \\
\text { Assuming } \\
6.5 \% \text { eff }\end{array}$ & $\begin{array}{c}\text { Burst } \\
\text { laser } \\
\text { communi } \\
\text { cations } \\
\text { power } \\
(\mathrm{W})\end{array}$ & $\begin{array}{c}\text { Data rate from } \\
\text { Alpha Centauri } \\
\text { during data } \\
\text { burst } \\
\text { Using reflector } \\
\text { for laser comm } \\
\text { (0.5\% duty } \\
\text { cycle) }\end{array}$ & $\begin{array}{c}\text { Average } \\
\text { Data } \\
\text { rate }\end{array}$ & $\begin{array}{c}\text { Beta } \\
\text { with } \\
\text { continue } \\
\mathrm{d}\end{array}$ & $\begin{array}{c}\text { Reach } \\
\text { Alpha } \\
\text { Centau } \\
\text { ri in } \\
\text { illumina } \\
\text { tion } \\
\text { 100yrs } \\
\text { (y/n) }\end{array}$ & $\begin{array}{c}\text { Reach } \\
\text { other } \\
\text { target } \\
\text { stars } \\
\text { (10lyrs } \\
(\mathrm{y} / \mathrm{n})\end{array}$ \\
\hline 0.001 & 0.85 & 0.005 & 1 & $37 \mathrm{kbs}$ & $0.2 \mathrm{kbs}$ & 0.24 & $\mathrm{y}$ & $\mathrm{y}$ \\
\hline 0.01 & 2.7 & 0.05 & 10 & $3.7 \mathrm{Mbs}$ & $20 \mathrm{kbs}$ & 0.14 & $\mathrm{y}$ & $\mathrm{y}$ \\
\hline 0.1 & 8.5 & 0.5 & 100 & $370 \mathrm{Mbs}$ & $2 \mathrm{Mbs}$ & 0.078 & $\mathrm{y}$ & $\mathrm{y}$ \\
\hline 1 & 27 & 5 & $1 \mathrm{~kW}$ & $37 \mathrm{Gbps}$ & $200 \mathrm{Mb}$ & 0.043 & $\mathrm{y}$ & $\mathrm{n}$ \\
\hline
\end{tabular}

Table 2. Reflector mass is equal to the base spacecraft mass. The reflector is used as the laser communication optics and is square with a thickness of 1 micron and has a density of $1.4 \mathrm{~g} / \mathrm{cc}$. We assume a $100 \mathrm{GW}$ laser for these numbers $(10 \mathrm{~km})$ on a side. $20 \%$ of bare craft mass is devoted to the RTG for electrical power. Burst data rate has a $0.5 \%$ duty cycle.

\section{Roadmap}

Should we begin on this path and if so how should we begin? Like any long journey it is easy get discouraged and not take the first steps. There are thousands of reasons not to begin. It is too hard, we are not technologically ready, we will not live to see the final journey to the stars... Most of these could be said about any profound endeavor. One difference on this journey is we have a very large scale of masses that are relevant to propel to extremely high speed rather than trying to propel a human and while one of the long term goals is to send a probe to a nearby star and return data, this is not the only objective. Part of the starting efforts will be to scope a more complete roadmap from desktop to orbital with an emphasis on understanding the TRL level of each element for future missions. Given the large range between our current chemical propellant propulsion and our goals of relativistic speeds and the range of useful masses from sub gram to large systems, we have an enormous parameter space to work in. All of these are along the path, particularly since this system is modular, scalable and on a very rapid development path and thus lends itself to a roadmap. With laser efficiencies near 50\% the rise in efficiency will not be one of the enabling elements along the road map but free space phase control over large distances during the acceleration phase will be. This will require understanding the optics, phase noise and systematic effects of our combined on-board metrology and off-board phase servo feedback. Reflector stability during acceleration will also be on the critical path as will 
increasing the TRL of the amplifiers for space use. For convenience we break the roadmap into several steps. One of the critical development items for space deployment is greatly lowering the mass of the radiators. While this sounds like a decidedly low tech item to work on, it turns out to be one of the critical mass drivers for space deployment. Current radiators have a mass to radiated power of 25 $\mathrm{kg} / \mathrm{kw}$, for radiated temperatures near $300 \mathrm{~K}$. This is an area where some new ideas are needed. With our current $\mathrm{Yb}$ fiber baseline laser amplifier mass to power of $5 \mathrm{~kg} / \mathrm{kw}$ (with a likely 5 year roadmap to $1 \mathrm{~kg} / \mathrm{kw}$ ) and current space photovoltaics of less than $7 \mathrm{~kg} / \mathrm{kw}$, the radiators are a serious issue for large-scale space deployment.

\section{Exploring the Interstellar Medium (ISM)}

On the development path to the nearest stars lays a wealth of information at the edge of and just outside our solar system. It is not "all or nothing" in going outside of our solar system. We will have many targets, including the solar system plasma and magnetic fields and its interface with the ISM, the heliopause and heliosheath, the Oort cloud outside and Kuiper belt inside, asteroids, KBO's, solar lens focus where the Sun acts as a gravitational lens to magnify distant objects. A more modest mission at $300 \mathrm{~km} / \mathrm{s}$ (60 AU/yr) would be wonderful for ISM studies.

We are looking for opportunities to leverage this effort into flight missions such as a CubeSat to test small sail acceleration as well as determine if ISS testing is feasible. Engaging both the public and private sector in mission concepts may well galvanize a larger community.

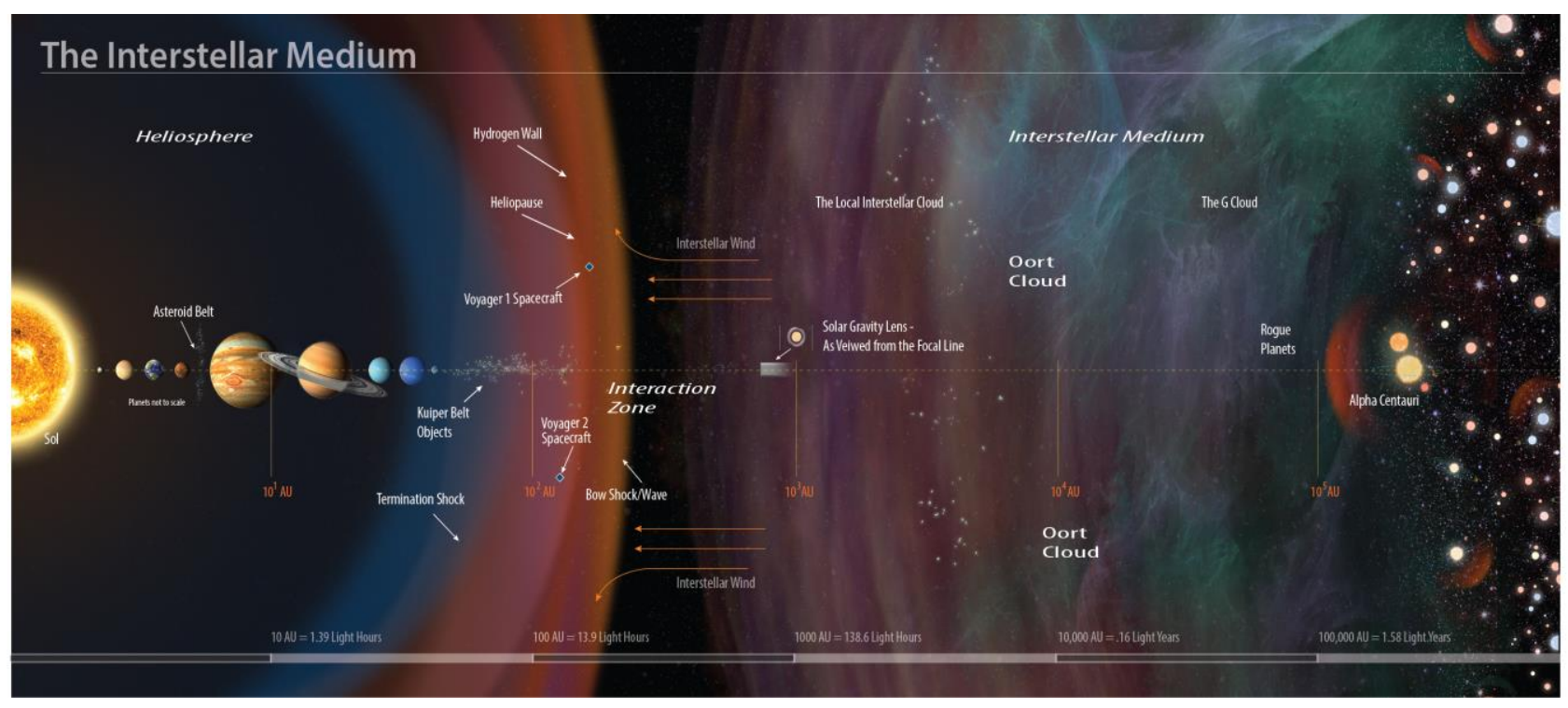

Figure 9. The Interstellar Medium and the Boundaries of the Heliosphere. [19]

\section{Other Benefits}

As we outline in our papers the same basic system can be used for many purposes including both stand-on and stand-off planetary defense from virtually all threats with rapid response, orbital debris mitigation, orbital boosting from LEO to GEO for example, future ground to LEO laser assisted launchers, standoff composition analysis of distant object through molecular line absorption, active illumination of asteroids and other solar system bodies, beamed power to distant spacecraft among others. The same system can also be used for beaming power down to the Earth via micro or $\mathrm{mm}$ 
waves for selected applications. This technology will give us transformative options that are not possible now and allows us to go far beyond our existing chemical propulsion systems.

\section{Acknowledgments}

We gratefully acknowledge the support and funding from the NASA NIAC 2015 NNX15AL91G and the NASA California Space Grant NASA NNX10AT93H in support of this research.

\section{Laser Sail - Non Relativistic solution See Lubin etal Roadmap to the Stars 2015 [2]}

\section{References}

[1] Bible, J., Bublitz, J., Johansson, I., Hughes, G.B., and Lubin, P. "Relativistic Propulsion Using Directed Energy," Nanophotonics and Macrophotonics for Space Environments VII, edited by Edward W. Taylor, David A. Cardimona, Proc. of SPIE Vol. 8876, 887605 (2013).

[2] Lubin, P., “A Roadmap to Interstellar Flight,” JBIS 2015.

[3] Lubin, P., Hughes, G.B., Bible, J., Bublitz, J., Arriola, J., Motta, C., Suen, J., Johansson, I., Riley, J., Sarvian, N., Clayton-Warwick, D., Wu, J., Milich, A., Oleson, M., Pryor, M., Krogen, P., Kangas, M., and O’Neill, H. "Toward directed energy planetary defense," Optical Engineering, Vol. 53, No. 2, pp 025103-1 to 025103-18 (Feb 2014), doi: 10.1117/1.OE.53.2.025103.

[4] Lubin, P., Hughes, G.B.J., Bible, J, Johansson Hummelgård, I., "Directed Energy for Planetary Defense and exploRation - Applications to Relativistic Propulsion and Interstellar Communications" edited by Gerald Cleaver - Journal of the British Interplanetary Society (JBIS) ( in press 2015)

[5] Lubin, P., Hughes, G.B. - invited chapter on "Directed Energy for Planetary Defense" - to be published in the Handbook of Cosmic Hazards and Planetary Defense - Springer Verlag book (in press 2015)

[6] Lubin, P., Hughes, G.B., Bible, J., Bublitz, J., Arriola, J., Motta, C., Suen, J., Johansson, I., Riley, J., Sarvian, N., Clayton-Warwick, D., Wu, J., Milich, A., Oleson, M., Pryor, M., Krogan, P. and Kangas, M. "Directed energy planetary defense (Plenary Paper)," Nanophotonics and Macrophotonics for Space Environments VII, edited by Edward W. Taylor, David A. Cardimona, Proc. of SPIE Vol. 8876, 887602 (Aug, 2013).

[7] Riley, J., Lubin, P., Hughes, G.B., O’Neill, H., Meinhold, P., Suen, J., Bible, J., Johansson, I., Griswold, J. and Cook, B. "Directed energy active illumination for near-Earth object detection," Nanophotonics and Macrophotonics for Space Environments VIII, edited by Edward W. Taylor, David A. Cardimona, Proc. of SPIE Vol. 9226 (Aug, 2014).

[8] Curtin, B., Bowers, J., "Thermoelectric power factor enhancement with gate-all-around silicon nanowires," Journal of Applied Physics 115, 143704 (2014); doi: 10.1063/1.4870962

[9] J. C. Hulme, J. K. Doylend, M. J. R. Heck, J. D. Peters, M. L. Davenport, J. T. Bovington, L. A. Coldren, and J. E. Bowers, "Fully integrated hybrid silicon free-space beam steering source with 32 channel phased array," An Invited Paper at the 2014 SPIE Conference

[10] Serway, R. and John Jewett. Physics for Scientists and Engineers with Modern Physics, Eighth Edition. Brooks/Cole, Cengage Learning, 2010. pp 471, 1011, 1118 
[11] Rowe, D.M., Thermoelectrics Handbook: Macro to Nano, CRC Press, 2009. 56-18.

[12] Vinod Chakka,Mohamed B. Trabia,Brendan O'Toole,Srujanbabu Sridharala,Samaan Ladkany, and Mostafiz Chowdhury. "Modeling and Reduction of Shocks on Electronic Components Within a Projectile" International Journal of Impact Engineering, vol. 35, pp. 1326-1338, 2008.

[13] Berman, Morris. "Electronic Components for High-g Hardened Packaging” 2006

[14] Bond, Alan, ed. "Project Daedalus: Target System Encounter Protection." Project Daedalus: The Final report on the BIS Starship Study. British Interplanetary Soc, 1978, S123-S125.

[15] Martin, Anthony R., ed. "Project Daedalus: Bombardment By Interstellar Material and Its Effects on the Vehicle." Project Daedalus: The Final report on the BIS Starship Study. British Interplanetary Soc, 1978, S116-S121.

[16] Zubrin, Robert, and A. Martin. "NIAC Study of the Magnetic Sail” (1999).

[17] Andrews, D.G. And ZUBRIN, R. "Magnetic Sails and Interstellar Travel." British Interplanetary Society, Journal 43 (1990): 265-272.

[18] Arora, Nitin. "Science Objectives," Jet Propulsion Laboratory / Caltech. Presented January 2015 at the Keck Institute for Space Studies Workshop for Science and Enabling Technologies to Explore the Interstellar Medium II.

[19] Interstellar Probe Exploring the Interstellar Medium and the Boundaries of the Heliosphere Report (2002), NASA/JPL kiss.caltech.edu/study/science/log\%20scale.jpg ium II. 